\title{
Predicting outcomes of recurrence after escalated-dose radiotherapy
}

Newly published research has characterized the natural history of biochemically recurrent disease in patients treated for localized prostate cancer using escalated-dose external beam radiotherapy (EBRT), and has identified risk factors for distant metastasis and prostate-cancer-specific mortality (PCSM).

Biochemical failure occurs in a proportion of patients treated with radical prostatectomy or radiotherapy. The progression of recurrent prostate cancer following biochemical failure is heterogeneous, and includes both indolent disease and aggressive recurrence. The natural history of recurrent prostate cancer following definitive treatment using radical prostatectomy has been well characterized, but less information is available on the outcomes following radiotherapy.

\section{4 ...patients with two} or more unfavourable risk factors had almost universally poor outcomes... 77

Dose-escalated EBRT, using a total radiation dose $\geq 75.6 \mathrm{~Gy}$, is the most common form of radiotherapy in use today. Zumsteg and colleagues considered 2,694 patients with localized prostate cancer who were treated with EBRT (total dose 75.6-86.4 Gy) between 1991 and 2008. 609 of these patients experienced biochemical failure, with a serum PSA measurement of $\geq 2 \mathrm{ng} / \mathrm{ml}$ above the PSA nadir. From the date of biochemical failure, the median time to distant metastases was 5.4 years, and that to PCSM was 10.5 years, resulting in a 5-year cumulative incidence of PCSM of $18 \%$.

Short PSA doubling time (PSADT $<3$ months), high initial tumour stage (T3b-T4) indicating extraprostatic tumour extension, high pretreatment Gleason score (8-10) and a short interval from the end of radiotherapy to biochemical failure (IBF $<3$ years) were all independent predictors for clinical progression following biochemical failure. Significant increases in the risk of clinical progression were observed with one of these risk factors (HR 2.36) and with two or more
(HR 5.67). In the 5 years after biochemical failure, patients with PSADT $\leq 3.2$ months had significantly higher risks of distant metastases (92.6\% versus 35.5\%, HR 5.09) and PCSM (34.4\% versus $11.7 \%$, HR 3.65) compared with patients with longer PSADTs. Similarly, patients with IBF $\leq 2.9$ years had increased 5-year risks of distant metastases (68.3\% versus $32.7 \%$, HR 2.68) and PCSM (28.3\% versus $11.1 \%$, HR 2.45), compared with patients with IBF $>2.9$ years. "In fact, patients with two or more unfavourable risk factors had almost universally poor outcomes, with more than $80 \%$ experiencing distant progression within 5 years of biochemical recurrence," explains corresponding author Michael Zelefsky. On this basis, the authors proposed a novel risk-stratification system for patients with biochemical failure after dose-escalated EBRT, placing patients with zero, one or two or more risk factors into groups of low, intermediate or high risk for distant progression, respectively.

The authors also questioned whether waiting until the PSA reaches $2 \mathrm{ng} / \mathrm{ml}$ above the post-treatment nadir is optimal for the timing of intervention. "We know from the postprostatectomy literature that the lower the post-treatment PSA is at the time of salvage radiotherapy, the more likely the patient is to benefit from this intervention," explains Zelefsky. "It is likely that the same is true for patients following definitive radiotherapy." Early identification of patients with persistent local disease using post-treatment biopsy or MRI could overcome the shortcomings of PSADT and IBF in this regard.

"Furthermore, novel molecular imaging techniques with greater sensitivity and specificity for detection of both local and metastatic prostate cancer could potentially revolutionize our ability to personalize treatment recommendations in the setting of biochemical recurrence," concludes Zelefsky.

\section{Robert Phillips}

Original article Zumsteg, Z. S. et al. The natural history and predictors of outcome following biochemical relapse in the dose escalation era for prostate cancer patients undergoing definitive external beam radiotherapy. Eur. Urol doi:10.1016/j.eururo.2014.09.028 